\title{
Genetic diversity of color phenotypes in the koi (Cyprinus carpio L.) as identified by molecular markers
}

\author{
Dani Bercovich ${ }^{1,2}$, Sigal Korem ${ }^{1,2}$, Lior Shauder $^{2}$, Gad Degani $^{2,3^{*}}$ \\ ${ }^{1}$ Human Molecular Genetics and Pharmacogenetics, MIGAL-Galilee Technology Center, Kiryat Shmona, Israel \\ ${ }^{2}$ Department of Biotechnology, Faculty of Life Sciences, Tel-Hai College, Upper Galilee, Israel \\ ${ }^{3}$ Aqua Agriculture Science Department, MIGAL-Galilee Technology Center, Kiryat Shmona, Israel; \\ *Corresponding author: gad@migal.org.il
}

Received 11 June 2012; revised 15 July 2012; accepted 24 July 2012

\begin{abstract}
The common carp (Cyprinus carpio) has a large variety of strains. The more popular are the koi (Japanese ornamental carp), which are still bred today to generate creative colors and patterns, giving rise to multiple phenotypes. Since koi are in great demand, there is a challenge to determine the genetics defining their quality. Two methods: 1) direct sequencing of five candidate gene regions, i.e., mitochondrial (cytochrome b, $12 S$ gene and the $D$-loop) and nuclear (red sensitive opsin and Rag-1) loci, to detect single nucleotide polymorphisms (SNP)s and 2) random amplification of polymorphic DNA (RAPD), were used to differentiate among four koi strains (Kohaku, Sanke, Ghost and Ohgon) and the common carp. Novel SNPs, distinguishing between koi and the common carp, were revealed in cytochrome $b$, the $D$-loop and in the red sensitive opsin; one was a missense mutation in cytochrome $b$ at position 15,860 , in which threonine in the common carp became alanine in all koi strains examined. The Kohaku strain was found to have two alleles in the mitochondrial fragments, forming two different haplotypes (subpopulations). These novel SNPs distinguished between koi strains and the common carp, and the RAPD method enabled further differentiation among the four koi strains.
\end{abstract}

Keywords: Koi; Skin Color; Polymorphism; SNP; RAPD

\section{INTRODUCTION}

The common carp (Cyprinus carpio) has a long history of domestication. Much artificial hybridization has been performed on this species over the years, leading to a large variety of strains in European and Asian countries $[1,2]$. Some of the carp strains generated over the years have been used as ornamental fish, because of the large variety in skin color and patterns. One of the more popular strains is the koi (Japanese ornamental carp). The koi fish are colored carp with much variation in color and patterns [3]. Some of the koi strains have been and are still being bred today in a specific manner, in order to generate creative colors and patterns, giving rise to multiple phenotypes among the strains existing today. The main demand today in Europe, the USA and East Asia, is for fish with an attractive and rare phenotype [4]. In a study on three main phenotypes, it was concluded that a gene complex, as opposed to a single gene, is responsible for the koi color $[5,6]$. These studies and others have found a correlation between the red phenotype and a microsatellite site, a specific allele correlated with red color [7]. Other groups have reported that at least five genes control the bright colors (white, yellow and orange) on the dark background of the wild type [8]. Genetic molecular markers are a useful tool in the identification of genetic polymorphisms and have contributed a lot to the research of intra and inter species polymorphisms, as well as to the building of phylogenetic trees at a high resolution [9]. The methods are divided into two main approaches: the use of random DNA-based genetic markers (such as random amplification of polymorphic DNA [RAPD], amplified fragment length polymorphism [AFLP] and microsatellites) and specific candidate gene single nucleotide polymorphism (SNP)s [10]. In this study, we used direct sequencing of candidate gene regions and RAPD. The candidate genes chosen were: two nuclear genes, red sensitive opsin and Rag-1, [11] and three mitochondrial genes, cytochrome $b, 12 S$ and the $D$-loop region of the mitochondrial DNA (mtDNA) [5]. The opsin protein is a photoreceptor with variable phenotypes, which are encoded by a few genes. The long-wave sensi- 
tive (LWS) gene was discovered to be polymorphic in guppy fish from different geographical areas and to have at least two copies in the genome [11]. Rag-1 encodes enzymes and is found in the VDJ recombination of immunoglobulins and in $\mathrm{T}$ cell receptors. An investigation on genetic intra-species polymorphism in the Mantella madagascariensis frog showed that 31 informative sites identified genetic changes among species, some of which are used today for the classification of the species of different strains [12].

MtDNA is known to be less conserved in evolution. Studies in vertebrates have shown that, since there are fewer mtDNA repair mechanisms, changes in mtDNA are more frequent. This is especially true in the D-loop, which is a noncoding sequence in mtDNA. However, this also occurs in the encoding genes, cytochrome $b$ and $12 S$ [13]. Another advantage of mtDNA is that it passes on maternal inheritance, and thus can trace complex heredity traits. MtDNA markers have been used a lot in research on sharks and eels. One study on mtDNA in the aquaculture field was carried out on guppy fish. A polymorphism of two nucleotides was found in cytochrome $b$ which serves as a molecular marker of strains [14]. The different colors and patterns in ornamental carp are thought to be a result of mutations in the wild carp, followed by crossbreeding between different strains. The goal of this study was to identify molecular markers that are linked to different koi strains, and thus could specifically identify strains. Such markers would be valuable in aqua agriculture.

\section{MATERIALS AND METHODS}

The fish were obtained from the Dan Fish Farm. Eighty five fish from five strains of koi and the common carp were randomly collected: 15 Sanke, 15 Kohaku, 15 Ghost, 20 Ohgon, 10 Ochiba and 10 common carp (Cyprinus carpio). In the second phase of the study, seven males and females from the Sanke, Kohaku, Ghost and Ohgon strains were collected for crossbreeding to generate F1. Larvae remained on the farm, until they were six weeks of age, when it was possible to clearly identify the phenotype. From the growing pools, twenty F1 offspring were sampled randomly, five individuals from each species for the remainder the koi fish were as followsKohaku: red and white; Sanke: black, white and red; $O$ chiba: gray; Ghost: orange and black; and Ohgon: white and orange (Figure 1).

DNA samples, which were derived from $20 \mathrm{mg}$ of tail fins, were extracted with a standard genomic DNA extraction kit. Mitochondrial DNA of four koi strains: Kohaku, Sanke, Ghost and Ohgon and the common carp, was amplified by polymerase chain reaction (PCR), followed by Sanger sequencing, using ABI 16 capillaries apparatus and primers, designed according to the Cypri- nus carpio complete mitochondrial genome, GenBank accession number: $\times 61010$. The fragments, which were amplified and sequenced, were from the mitochondrial genes: cytochrome $b, 12 S$ and $D$-loop and the nuclear genes: red sensitive opsin and Rag-1, GenBank accession numbers: AB055656 and AY787040, respectively.

RAPD analysis was performed on five koi strains: Kohaku, Sanke, Ghost, Ochiba and Ohgon. Eight primers were designed, and among them, two (opa-3, opa-9) were chosen; they showed the clearest patterns of the different haplotypes, enabling of the research. The color phenotypes of differentiation among the strains.

\section{RESULTS}

In order to evaluate the genetic distance between the common carp and koi, mtDNA fragments from cytochrome $b, 12 S$ and the $D$-loop (control region) genes were sequenced. Thirteen SNPs were found in a fragment of 356 base pairs (bp) from the cytochrome $b$ gene; three of which were missense mutations (p.Thr190Ala c.A15860G; p.Val192Ala c.T15867C; p.Ile218Val c.A15944G). Five of the SNPs, which included the Thr190Ala missense mutation, distinguished between the common carp and the four koi strains (Table 1 and Figure 1). The other eight SNPs were identical in the carp and the other strains, with the exception of the Kohaku strain, which comprised two subpopulations, one with the common allele (like in the other strains and the carp) and the second with a polymorphic allele. We named it the "rare allele”, since it was found only in 33\% of the Kohaku strains and not in any other strain. These data enabled us to draw a phylogenetic tree based on the cytochrome $b$ polymorphism, which implied that the koi "rare allele" strain branched before the separation between the common carp and the other koi strains (Figure 2). A cross between the Kohaku "rare allele" female and a Sanke male generated F1 offspring with both koi phenotypes and the "rare allele" genotype, indicating that this mtDNA sequence was maternally inherited.

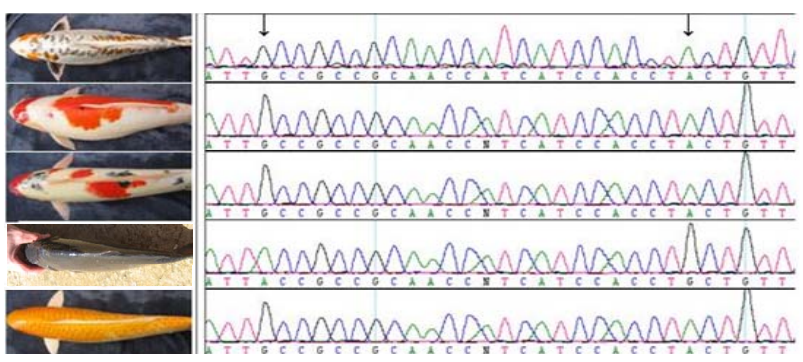

Figure 1. An example of a sequence comparison between the common carp and koi strains at positions 15860 and 15883 on the cytochrome $b$. Each sequence represents data obtained from individuals of the same strain. Only two of the five SNPs are shown. The order from top to bottom: Ghost, Kohaku, Sanke, C. carpio and Ohgon. 


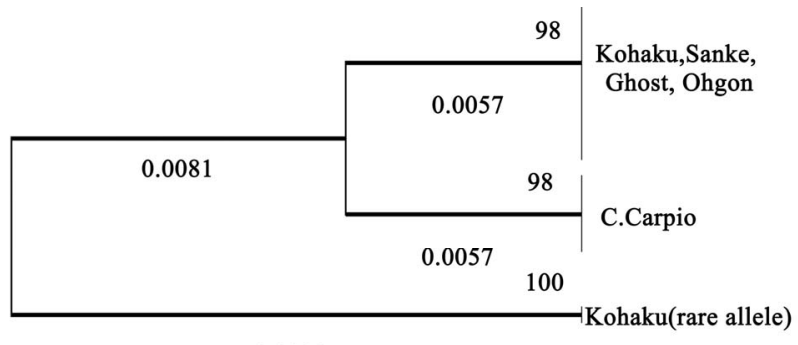

0.0138

Figure 2. A phylogenetic tree based on cytochrome $b$ polymorphisms. The sum of the distances from the optimal tree was 0.00334 . The bootstrap data are based on 1000 repeats presented as a percentage on each branch .The phylogenetic distances were calculated according to the "Maximum Composite Likelihood" [15]. The database from which this tree was built is based on 356 positions. The "Kohaku, Sanke, Ghost, Ohgon" branch includes 25 individuals; the " $C$. carpio" branch is from ten individuals; and the "Kohaku (rare allele)" branch is from two individuals.

Table 1. Haplogroups of cytochrome b in the koi and common carp.

\begin{tabular}{|c|c|c|c|c|c|c|c|c|c|c|c|c|c|}
\hline \multirow{2}{*}{ Strain } & \multicolumn{13}{|c|}{ SNPs } \\
\hline & 1 & 2 & 3 & 4 & 5 & 6 & 7 & 8 & 9 & 10 & 11 & 12 & 13 \\
\hline Kohaku (wt) & $\mathrm{T}$ & G & $\mathrm{C}$ & A & G & G & $\mathrm{T}$ & A & $\mathrm{T}$ & A & $\mathrm{T}$ & C & G \\
\hline $\begin{array}{c}\text { Kohaku } \\
\text { (rare allele) }\end{array}$ & C & G & $\mathrm{T}$ & A & A & A & C & G & $\mathrm{C}$ & A & $\mathrm{C}$ & $\mathrm{C}$ & G \\
\hline Sanke (wt) & $\mathrm{T}$ & G & C & A & G & G & $\mathrm{T}$ & A & $\mathrm{T}$ & A & $\mathrm{T}$ & $\mathrm{C}$ & G \\
\hline Ghost (wt) & $\mathrm{T}$ & G & C & A & G & G & $\mathrm{T}$ & A & $\mathrm{T}$ & A & $\mathrm{T}$ & $\mathrm{C}$ & G \\
\hline Ohgon (wt) & $\mathrm{T}$ & G & C & A & G & G & $\mathrm{T}$ & A & $\mathrm{T}$ & A & $\mathrm{T}$ & C & G \\
\hline C. carpio & $\mathrm{T}$ & A & $\mathrm{C}$ & G & G & G & $\mathrm{T}$ & A & $\mathrm{T}$ & G & $\mathrm{T}$ & $\mathrm{T}$ & A \\
\hline
\end{tabular}

Thirteen SNPs from the cytochrome $b$ gene are shown: c15856t, a15860g, t15867c, a15883g, a15944g, a15958g, t15982c, a15985g, c16006t, a16024g, t16081c, c16156t and a16189g. The Kohaku strain was divided into two subpopulations, with eight polymorphic sites (shaded in grey in the "rare allele" strain) separating between a "rare allele" strain that was discovered in a few individuals and the other Kohaku individuals. In the Sanke, Ghost and Ohgon strains, only the WT allele was found. Five SNPs distinguished the common carp (C. carpio) from the koi strains (shaded in grey in the $C$. carpio strain).
The purpose of generating offspring (F1) from Sanke, Kohaku, Ghost and Ohgon strains was that the Kohaku strain is generally cross briding with the Sanke strain, and because we found a rare allele in the Kohaku strain only, we wanted to know if the origin of this rare allele is from the Sanke strain. The Sanke strain has a red, white and black colors and the Kohaku in red and white. So the cross was to see if the black color in the rare allele came from the Sanke. The cross between the Ghost to Ghost and between the Ohgon and Ohagon (generating F1 offspring's), was as a control briding step only, and so we did not use the Ochiba and common carp as control as well.

However, this polymorphism did not distinguish between the two koi strain colors. Four SNPs in a 199 bp fragment from the mtDNA $12 S$ gene were detected in all Kohaku strains. Thus, none of these SNPs were informative, since they were not able to differentiate between the common carp and the koi strains.

In a $380 \mathrm{bp}$ fragment from the mtDNA $D$-loop region, 15 SNPs were found. Ten were only in the Kohaku strain, and five others demonstrated a significant difference between the koi and the common carp (Table 2 and Figure 3). These results were used to build a phylogenetic tree based on the $D$-loop polymorphism, which showed that the Kohaku with the "rare allele" is the most genetically distant, when compared to the other strains examined (Figure 4). A cross between the Kohaku D-loop "rare allele" female and a Sanke male generated F1 offspring with both koi phenotypes and the "rare allele" genotype, indicating that this mtDNA sequence was maternally inherited. However, this polymorphism did not distinguish between the two koi strain colors.

Two nuclear genes, the Rag-1 and red sensitive opsin, were also screened for SNPs. In the red sensitive opsin gene, a 458 bp fragment from the end of the coding region and the 3' UTR were sequenced. The three SNPs (two in the coding region (p.Val328Val c.1076A > T; p.Ser342Ser c.1118T > C) and one in 3' UTR, (c.1350G > A) (Table 3), were found to form five different haplotypes. The

Table 2. The haplogroups of the D-loop in the koi and common carp.

\begin{tabular}{|c|c|c|c|c|c|c|c|c|c|c|c|c|c|c|c|}
\hline \multirow{2}{*}{ Strains } & \multicolumn{15}{|c|}{ SNPs } \\
\hline & 1 & 2 & 3 & 4 & 5 & 6 & 7 & 8 & 9 & 10 & 11 & 12 & 13 & 14 & 15 \\
\hline Kohaku (wt) & G & G & $\mathrm{C}$ & A & A & A & A & $\mathrm{T}$ & $\mathrm{T}$ & G & G & G & $\mathrm{C}$ & $\mathrm{C}$ & $\mathrm{T}$ \\
\hline Kohaku (rare allele) & A & A & $\mathrm{T}$ & $\mathrm{C}$ & G & A & G & $\mathrm{C}$ & $\mathrm{C}$ & G & A & G & $\mathrm{C}$ & $\mathrm{T}$ & $\mathrm{C}$ \\
\hline Sanke $(w t)$ & G & G & $\mathrm{C}$ & A & A & A & A & $\mathrm{T}$ & $\mathrm{T}$ & G & G & G & $\mathrm{C}$ & $\mathrm{C}$ & $\mathrm{T}$ \\
\hline Ghost (wt) & G & G & $\mathrm{C}$ & A & A & A & A & $\mathrm{T}$ & $\mathrm{T}$ & G & G & G & $\mathrm{C}$ & $\mathrm{C}$ & $\mathrm{T}$ \\
\hline Ohgon (wt) & G & G & $\mathrm{C}$ & A & A & A & A & $\mathrm{T}$ & $\mathrm{T}$ & G & G & G & $\mathrm{C}$ & $\mathrm{C}$ & $\mathrm{T}$ \\
\hline C. carpio & G & G & C & A & A & G & A & $\mathrm{T}$ & $\mathrm{T}$ & $\mathrm{T}$ & $\mathrm{G}$ & A & $\mathrm{T}$ & $\mathrm{T}$ & $\mathrm{T}$ \\
\hline
\end{tabular}

Fifteen SNPs from the D-loop are shown: a266g, 1290g, t291c, c300a, g313a, a321g, g333a, a466g, a483g, t489c, t491c and c517t. The Kohaku strain was divided into two subpopulations, wild type Kohaku (wt) and "rare allele" Kohaku, as ten polymorphic sites were detected in it (shaded in grey in the rare allele strain). In the Sanke, Ghost and Ohgon, only the WT allele was found, except for a SNP at position 466, in two individuals, one Sanke and one Ohgon. Five polymorphisms distinguished the common carp (C. carpio) from the koi strains (shaded in grey in the C. carpio strain). 
Table 3. SNPs found in the red sensitive the opsin nuclear gene in four koi strains and common carp.

\begin{tabular}{|c|c|c|c|c|c|c|}
\hline Strains & & Koi (ornal & ntal carp) & & Common Carp & \\
\hline Sample size & Kohaku 13 & Sanke 8 & Ghost 9 & Ohgon 7 & C. carpio 10 & \\
\hline $\begin{array}{l}\text { DNA and protein } \\
\mathrm{AA} / \mathrm{AB} / \mathrm{BB} \text { alteration }\end{array}$ & \multicolumn{5}{|c|}{ Ratio of DNA alteration } & Remarks \\
\hline p.Val328Val c.1076A > T & $5 / 1 / 7$ & $4 / 0 / 4$ & $5 / 3 / 1$ & $2 / 1 / 4$ & $10 / 0 / 0$ & \\
\hline p.Ser342Ser c.1118T > C & $7 / 1 / 5$ & $3 / 1 / 4$ & $2 / 3 / 4$ & $1 / 2 / 4$ & $10 / 0 / 0$ & Encoded \\
\hline c. $1350 \mathrm{G}>\mathrm{A}$ & $3 / 1 / 9$ & $2 / 1 / 5$ & $1 / 0 / 8$ & $1 / 0 / 6$ & $10 / 0 / 0$ & 3' UTR \\
\hline
\end{tabular}

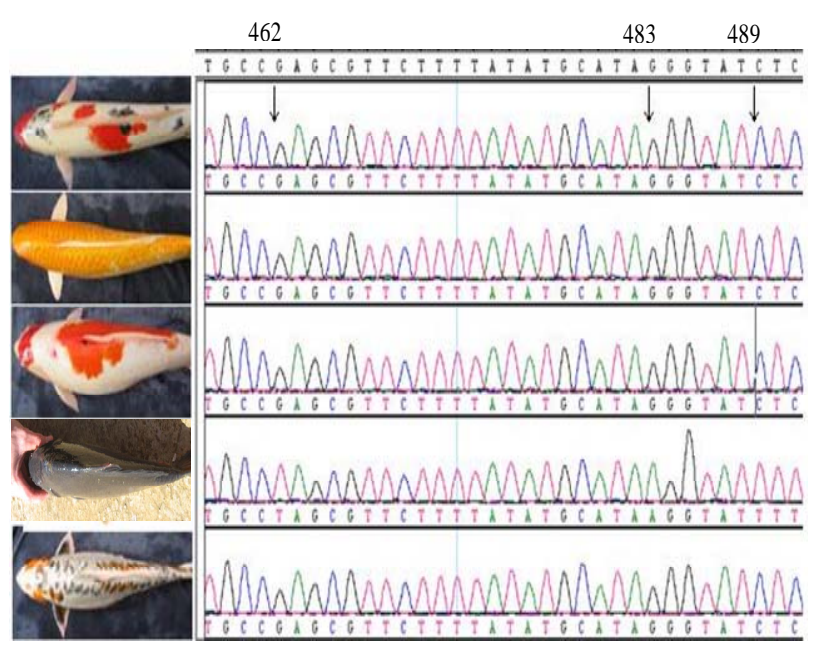

Figure 3. An example of a sequence comparison between the common carp and koi strains at positions 483, 462 and 489 on the $D$-loop. Each sequence represents data obtained from all individuals of the same strand. The SNPs are at positions 462, 483 and 489. The order of the strains from top to bottom is: Sanke, Ohgon, Kohaku, C. carpio and Ghost.

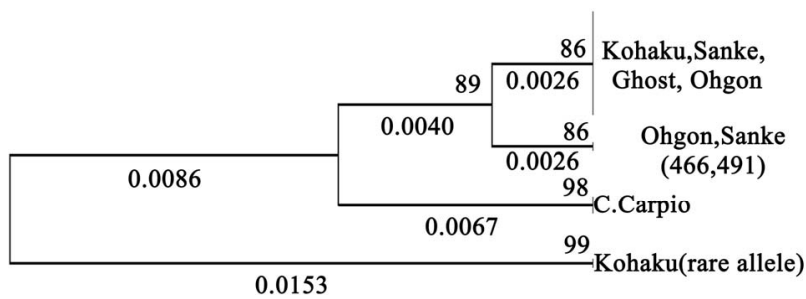

Figure 4. A phylogenetic tree based on D-loop polymorphisms. The sum of the distances from the optimal tree was 0.0398 . The bootstrap data are based on 1000 repeats presented as a percentage on each branch [16]. The phylogenetic distances were calculated according to the "Maximum Composite Likelihood" [15]. The database for building this tree was based on 380 positions. The "Kohaku, Sanke, Ghost, Ohgon" branch included 23 individuals; "the Ohgon, Sanke" one from two individuals; the "C. carpio" branch was from four individuals; and the "Kohaku (rare allele)” branch was from two individuals. different koi haplotypes were heterozygous in all three positions, 1076, 1118 and 1350, whereas the common carp was homozygous; A/A in position 1076, T/T in 1118 and G/G in 1350 (data not shown). In the Rag-1 gene, a fragment of $154 \mathrm{bp}$ was sequenced and seven SNPs were identified, which generated nine different haplotypes in the four different koi strains and common carp. However, since there was no correlation between the haplotypes and the strains, these SNPs could not distinguish between inter or intra strain differences (data not shown). A phylogenetic tree based on the red sensitive opsin, cytochrome $b$ and opsin polymorphisms distinguished the common carp from the koi strains (Figure 5).

The second approach was to screen differences and polymorphisms randomly, instead of according to special candidate genes. This was carried out by the random amplification of polymorphic DNA (RAPD) assay with two

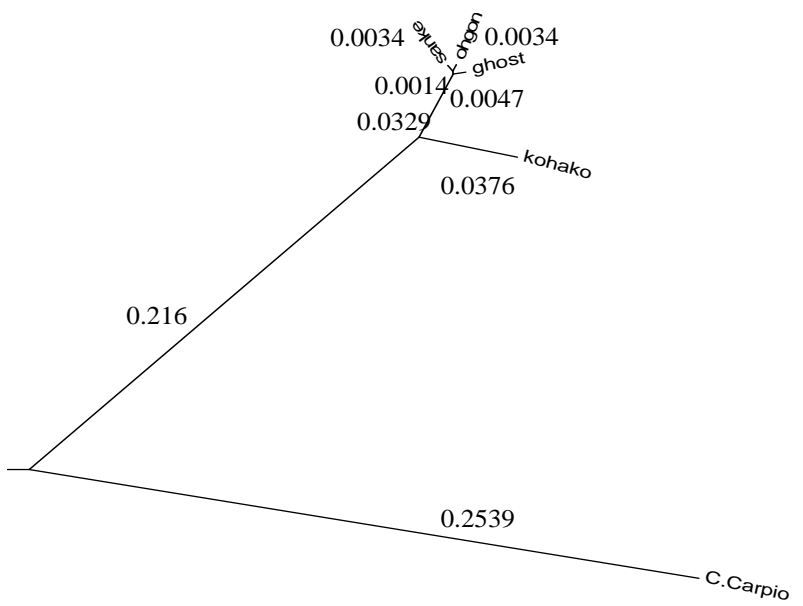

Figure 5. Phylogenetic tree based on red sensitive opsin, cytochrome $b$ and D-loop polymorphisms. The data from 31 SNPs found in the red sensitive opsin, cytochrome $b$ and D-loop genes were calculated by the UPGMA method [17]. The sum of the distances of the branches from the optimal tree was 0.553 , as calculated by Nei's original genetic distance [18]. 
primers, opa-3 and opa-9. Amplification with the opa-3 primer revealed nine fragments, producing 23 different haplotypes (Figure 6). With the opa-9 primer, the nine resulting fragments gave 28 different haplotypes. When these 18 fragments were analyzed by means of a phylogenetic tree, two main branches were obtained, one with the Ghost and Ohgon strains and another, which subdivided into two more branches, one including Kohaku and Sanke and the other with the Ochiba strain (Figure 7).

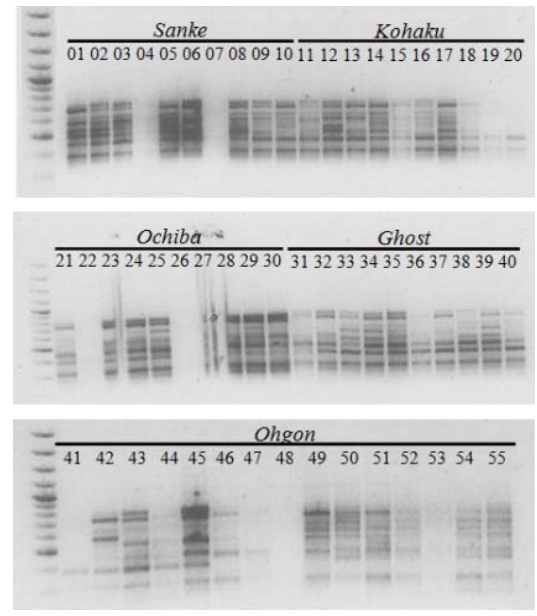

Figure 6. Gel electrophoresis of RAPD PCR with the opa-3 primer in five koi strains.

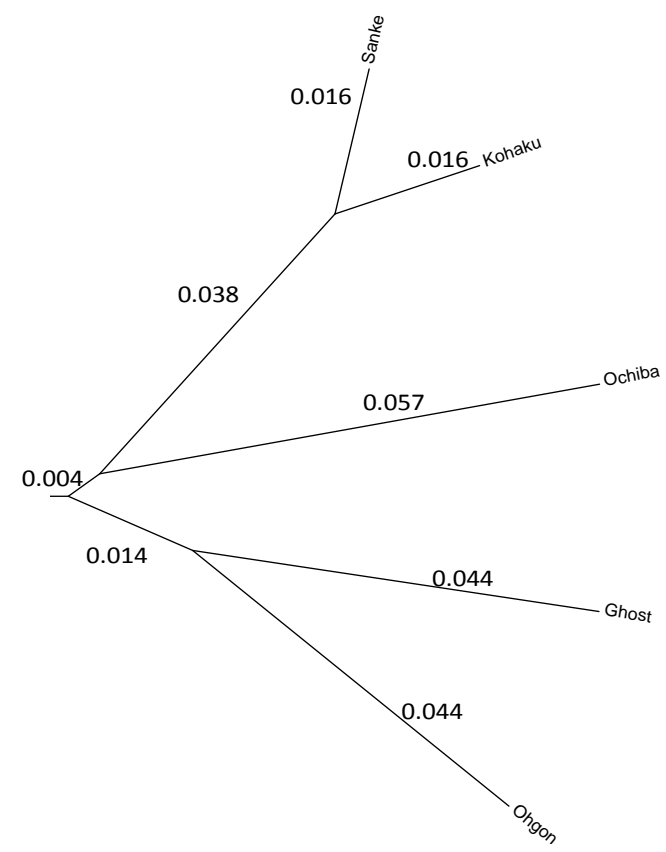

Figure 7. A phylogenetic tree based on a RAPD PCR with opa-3 and opa-9 primers. The data was obtained from 18 RAPD fragments, which were amplified by opa-3 and opa-9 primers. The data was calculated by the UPGMA method. The sum of the distances of the branches from the optimal tree was 0.2332 , as calculated by Nei's original genetic distance [18].

\section{DISCUSSION}

In this study, an attempt was made to identify genetic differences among four strains of koi and the common carp, using SNPs on three mitochondrial and two nuclear genes and basic RAPD technology. Genetic relationships among six populations of domesticated carp (Cyprinus carpio), a species with a partially sequenced genome, has been carried out using genetic markers, such as $18 \mathrm{~S}$ and RAPD, as well as SNPs [3]. From the five different koi strains, Kohaku and Sanke are known to be more crossbred because of their color demand, and as a result, harbor more genetic polymorphisms and are more genetically related [3]. In our study, we also found multiple genetic polymorphisms in the mtDNA of the Kohaku strain. According to the RAPD method, these two strains are genetically closer.

The assumption that koi fish are descended from the common carp has existed for a long time [19]. Several studies over the years have engaged in detecting genetic differences among strains of different carp and koi species, revealing phylogenetic relationships [3,6,14,20,21]. The present project has contributed more information towards its completion, by focusing on genetic regions, which indicate clear, but close, phylogenetic distances between the common carp and koi strains.

Since mtDNA is less stable than nuclear DNA in acquiring new mutations [22], we chose these two types of DNA regions. In this study, differences in two mitochondrial genes (cytochrome $b$ and D-loop) and in one nuclear one, the red sensitive opsin, were discovered. These novel SNPs distinguished the common carp from the koi, but did not differentiate among the koi strains. In the red sensitive opsin nuclear gene, three SNPs were found to be heterozygous (or rarely homozygous) only in the koi strains and not in the common carp (Table 3).

Genetic polymorphism has been detected in the mtDNA of guppy strains [5]. In the present study, we found several novel SNPs in the three regions tested in the mtDNA, five novel SNPs in the cytochrome $b$ gene (one of which is a missense mutation; Thr190Ala) and five other novel SNPs in the D-loop region, which clearly segregate the koi strains from the common carp. Thus, these are good genetic markers, which can be utilized for distinguishing between the koi and the common carp, even in very early stages of fish development.

We used the three genomic regions (cytochrome $b$, D-loop and red sensitive opsin) that were found to harbor genetic polymorphisms, as a basis for genetic variance between the koi strains and the common carp. The Anova test for genetic variance was executed twice, once on the koi strain data alone, and a second time, when the data on the common carp was included. Omission of the common carp from the Anova analysis decreased the genetic variance and the Fst value, which was 0.221 (21\%, P < 
0.05), in comparison to a Fst value of 0.342 (34\%, $\mathrm{P}<$ 0.01 ), when the common carp was included. When this test was applied to the RAPD data, based on the genetic polymorphism obtained from the opa- 3 and opa- 9 primers "Figure 6", the Fst value was $0.183(18 \%, \mathrm{P}=0.01)$. According to this type of analysis, the SNPs revealed higher genetic variations between the koi strains and the common carp, in comparison to the RAPD, but this finding is probably affected more by the polymorphism found in the red sensitive opsin gene and less by the mtDNA regions, where we found less genetic variance and which was mostly due to the Kohaku strain. The RAPD analysis showed more genetic similarity between the Kohaku and the Sanke strains, in comparison to the other koi strains. This is in agreement with previous findings by Jackson et al. [6].

In the Kohaku strain, two different alleles were identified in the three mitochondrial fragments; thus, forming two different haplotypes, which differed in two amino acids in the cytochrome $b$ gene: V192A and I218V. Apparently, this is a subpopulation of the Kohaku. This is based on the phylogenetic tree of the cytochrome $b$ polymorphism, which shows that the Kohaku "rare allele" strain probably branched before the separation between the common carp and the other koi strains "Figure 2".

In summary, by means of candidate gene sequencing, we identified novel SNPs, which can distinguish between the four koi strains and the common carp, but not among the different koi strains. However, the RAPD method was able to distinguish among the koi strains studied here, as well as between them and the common carp.

\section{REFERENCES}

[1] Flajšhans, M. and Hulata, G. (2006) Common carp Cyprinus carpio. Genimpact final scientific report, University of South Bohemia, Vodnany, Czech Republic. In: Agricultural Research Organization, Bet Dagan, 32-39.

[2] Haynes, G.D., Gilligan, D.M., Grewe, P. and Nicholas, F.W. (2009) Population genetics and management units of invasive common carp Cyprinus carpio in the MurrayDarling Basin, Australia. Journal of Fish Biology, 75, 295-320. doi:10.1111/j.1095-8649.2009.02276.x

[3] David, L., Rosenberg, N.A., Lavi, U., Feldman, M.W. and Hillel, J. (2007) Genetic diversity and population structure inferred from the partially duplicated genome of domesticated carp, Cyprinus carpio L. Genetics Selection Evolution, 39, 319-340. doi:10.1186/1297-9686-39-3-319

[4] Perelberg, A., Ronen, A., Hutoran, M., Smith, Y. and Kotler, M. (2005) Protection of cultured Cyprinus carpio against a lethal viral disease by an attenuated virus vaccine. Vaccine, 23, 3396-3403. doi:10.1016/j.vaccine.2005.01.096

[5] Degani, G. (2004) Application of RAPD and cytochrome $b$ sequences to the study of genetic variations in F1 and F2 generations of two different strains of guppy (Poecilia reticulata, Peter 1854). Aquaculture Research, 35, 807815. doi:10.1111/j.1365-2109.2004.01071.x

[6] Jackson, K., Goldberg, D., Yehuda, Y. and Degani, G. (2000) Molecular DNA variation in koi (Cyprinus carpio) of various color patterns. Israeli Journal of Aquaculture-Bamidgeh, 3, 151-158.

[7] David, L., Rothbard, S., Rubinstein, I., Katzman, H., Hulata, G., Hillel, J. and Lavi, U. (2004) Aspects of red and black color inheritance in the Japanese ornamental (Koi) carp (Cyprinus carpio L.). Aquaculture, 233, 129-147.

[8] Katasonov, V.Y. (1978) A study of pigmentation in hybrids between the common carp and decorative Japanese carp: III. The inheritance of blue and orange patterns of pigmentation. Genetika, 14, 2184-2192.

[9] Khoo, G., Lim, K.F., Gan, D.K., Chen, F., Chan, W.K., Lim, T.M. and Phang, V.P. (2002) Genetic diversity within and among feral populations and domesticated strains of the guppy (Poecilia reticulata) in Singapore. Marine Biotechnology, 4, 367-378. doi:10.1007/s10126-002-0007-z

[10] Shen, X., Yang, G., Liu, Y., Liao, M., Wang, X., Zhu, M., Song, W., Zou, G., Wei, Q., Wang, D. and Chen, D. (2007) Construction of genetic linkage maps of guppy (Poecilia reticulata) based on AFLP and microsatellite DNA markers. Aquaculture, 271, 178-187. doi:10.1016/j.aquaculture.2007.06.003

[11] Hoffmann, M., Tripathi, N., Henz, S.R., Lind-holm, A.K., Weigel, D., Breden, F. and Dreyer, C. (2007) Opsin gene duplication and diversification in the guppy, a model for sexual selection. Proceedings of the Royal Society BBiological Sciences, 274, 33-42. doi:10.1098/rspb.2006.3707

[12] Chiari, Y., Vences, M., Vieites, D.R., Rabemananjara, F., Bora, P., Ramilijaona Ravoahangimalala, O. and Meyer A. (2004) New evidence for parallel evolution of colour patterns in Malagasy poison frogs (Mantella). Molecular Ecology, 13, 3763-3774. doi:10.1111/j.1365-294X.2004.02367.x

[13] Benzie, J.A., Ballment, E., Forbes, A.T., Demetriades, N.T., Sugama, K., Haryanti and Moria S. (2002) Mitochondrial DNA variation in Indo-Pacific populations of the giant tiger prawn, Penaeus monodon. Molecular Ecology, 11, 2553-2569. doi:10.1046/j.1365-294X.2002.01638.x

[14] Wang, C.-H. and Li, S.-F. (2004) Phylogenetic relationships of ornamental (koi) carp, Oujiang color carp and Long-fin carp revealed by mito-chondrial DNA COII gene sequences and RAPD analysis. Aquaculture, 231, 83-91. doi:10.1016/j.aquaculture.2003.10.040

[15] Tamura, K., Nei, M. and Kumar, S. (2004) Prospects for inferring very large phylogenies by using the neighborjoining method. Proceedings of the National Academy of Sciences USA, 101, 11030-11035. doi:10.1073/pnas.0404206101

[16] Felsenstein, J. (1985) Confidence limits on phylogenies: An approach using the bootstrap. Evolution, 39, 783-791. doi:10.2307/2408678

[17] Sneath, P.H. and Sokal, R.R. (1973) Numerical Taxonomy. Freeman, San Francisco. 
[18] Nei, M. and Li, W.H. (1979) Mathematical model for studying genetic variation in terms of restriction endonucleases. Proceedings of the National Academy of Sciences USA, 76, 5269-5273. doi:10.1073/pnas.76.10.5269

[19] Taniguchi, N., Kijima, A., Tamura, T., Takegami, K. and Yamasaki, I. (1986) Color, growth and maturation in ploidy-manipulated fancy carp. Aquaculture, 57, 321-328. doi:10.1016/0044-8486(86)90210-3

[20] Kohlmann, K., Gross, R., Murakaeva, A. and Kersten, P. (2003) Genetic variability and structure of common carp (Cyprinus carpio) populations throughout the distribution range inferred from allozyme, microsatellite and mitochondrial DNA markers. Aquatic Living Resources, 16, 421-431. doi:10.1016/S0990-7440(03)00082-2

[21] Kohlmann, K. and Kersten, P. (1999) Genetic variability of German and foreign common carp (Cyprinus carpio L.) populations. Aquaculture, 173, 435-445. doi:10.1016/S0044-8486(98)00474-8

[22] Sigurgardottir, S., Helgason, A., Gulcher, J.R., Stefansson, K. and Donnelly, P. (2000) The mutation rate in the human mtDNA control region. American Journal of Human Genetics, 66, 1599-1609. doi:10.1086/302902 